\title{
Acrylic Foreign Body in the Orbit : A Multidisciplinary Approach
}

https://doi.org/10.47210/bjohns.2020.v28i3.276

Nikhil Rajendra Dhorje, ${ }^{1}$ Kshitij Dhaval Shah, ${ }^{1}$ Madhusudhan $V^{1}$ Pushkaraj Abhay Kulkarni, ${ }^{1}$ Renuka Anil Bradoo ${ }^{1}$

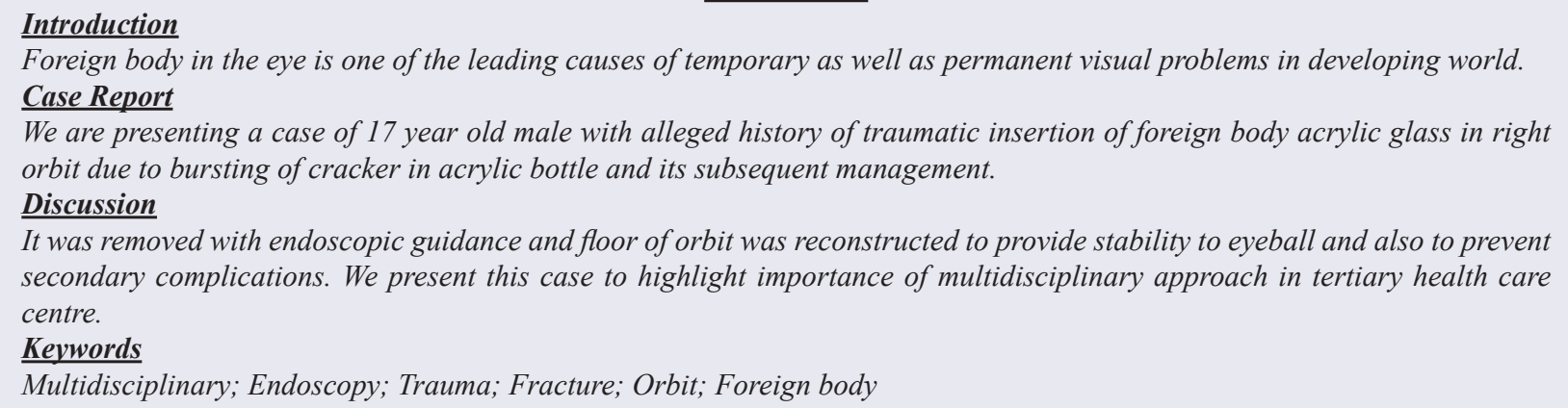

$\mathrm{F}$ oreign body in the eye is one of the leading causes of temporary as well as permanent visual problems in developing world. According to reports, maximum numbers of patients are seen in 21 to 40 years of age group with bimodal peak between March and November, with male predominance. Sharp pointed object related injuries are most common in work related conditions. ${ }^{1}$ We are presenting a case of 17 year old male with history of traumatic insertion of foreign body acrylic glass in right orbit due to bursting of cracker in acrylic bottle and its subsequent management.

\section{Case Report}

17 year old male patient came in casualty with a piece of acrylic in right orbit as a result of accidental trauma due to bursting of cracker in an acrylic bottle.

1 - Department of ENT, Lokmanya Tilak Municipal Medical College and General Hospital Sion, Mumbai

\section{Corresponding author:}

Dr Nikhil Rajendra Dhorje

email: nikhildhorje43@gmail.com

On examination, the patient was conscious and well oriented to surroundings with right eyeball pressed downwards due to foreign body with no apparent rupture of the globe. (Fig. 1) There was enophthalmos of right eye due to downward pushing of eyeball with resultant floor of orbit fracture as a result of presence of foreign body in right orbit, with no apparent phthisis bulbi. The right upper eyelid was injured, the right lower eyelid was rolled inwards along with the foreign body, and the medial canthus on right was injured along with some injury near dorsum of nose while the right lateral canthus and right upper eyebrow was intact. Left eye was normal.

Ophthalmological consultation was taken, visual assessment and extraocular movements was not possible on right side as the eyeball was pushed below into maxillary cavity by rupturing the floor of orbit. The exact cause of loss of extraocular movements on right side. was it because of entrapment and/or injury to fourth (IV) and sixth (VI) cranial nerve were not possible to assess due to presence of foreign body in right orbit pushing the eyeball downwards. A computed tomography (CT) scan of orbit, paranasal sinuses (PNS) and brain was done to assess the three dimensional anatomical orientation 


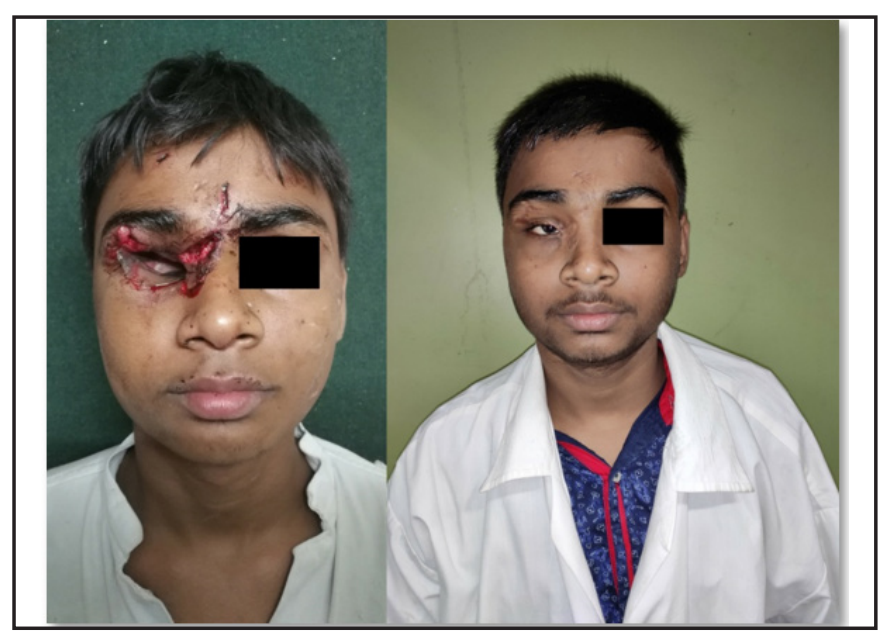

Fig. 1. On left, preoperative photograph of patient with foreign body in situ in right orbit. On right, postoperative photograph of patient at time of discharge.

of foreign body, extent of damage to surrounding structures and to help in planning the removal of body. CT scan was suggestive of downward displacement of right eyeball into maxillary sinus with fracture of floor of orbit, without rupture of eyeball with no optic nerve damage. (Fig. 2) Plastic surgery consultation was taken for right upper eyelid and floor of orbit reconstruction. Pneumocephalus was also noted on right side which was managed conservatively after taking neurosurgical consultation.

As it was a case of acute accidental trauma, the patient was taken for removal of foreign body immediately after arrival in hospital under general anaesthesia, with neurosurgeons on standby. Firstly, the foreign body was removed from orbit (Fig. 3), followed by reconstruction of right upper eyelid.

Right upper eyelid reconstruction was done by primarily closing the defect. Peritomy was performed, in which an incision was made at the limbus in order to reflect the conjunctiva and Tenon's capsule of eye to expose the sclera and extraocular muscles to carry out forced duction tests on superior, inferior, medial and lateral rectus muscles. The forced duction tests on all four rectii were normal. A small iris sphincter tear was also noted medially. Pupillary reaction could not be checked due to patient being under anaesthesia. As the visual prognosis of the patient was guarded, decision was taken to temporarily reconstruct the floor of orbit. Endoscopically right sided uncinectomy and middle meatal antrostomy was done, maxillary sinus ostium opened. A 16 FG Foleys balloon catheter was introduced into maxillary sinus and was inflated with around $8 \mathrm{cc}$ of saline. There was immediate elevation of the earlier depressed right eyeball after inflation of balloon. Immediate postoperative CT scan of the patient

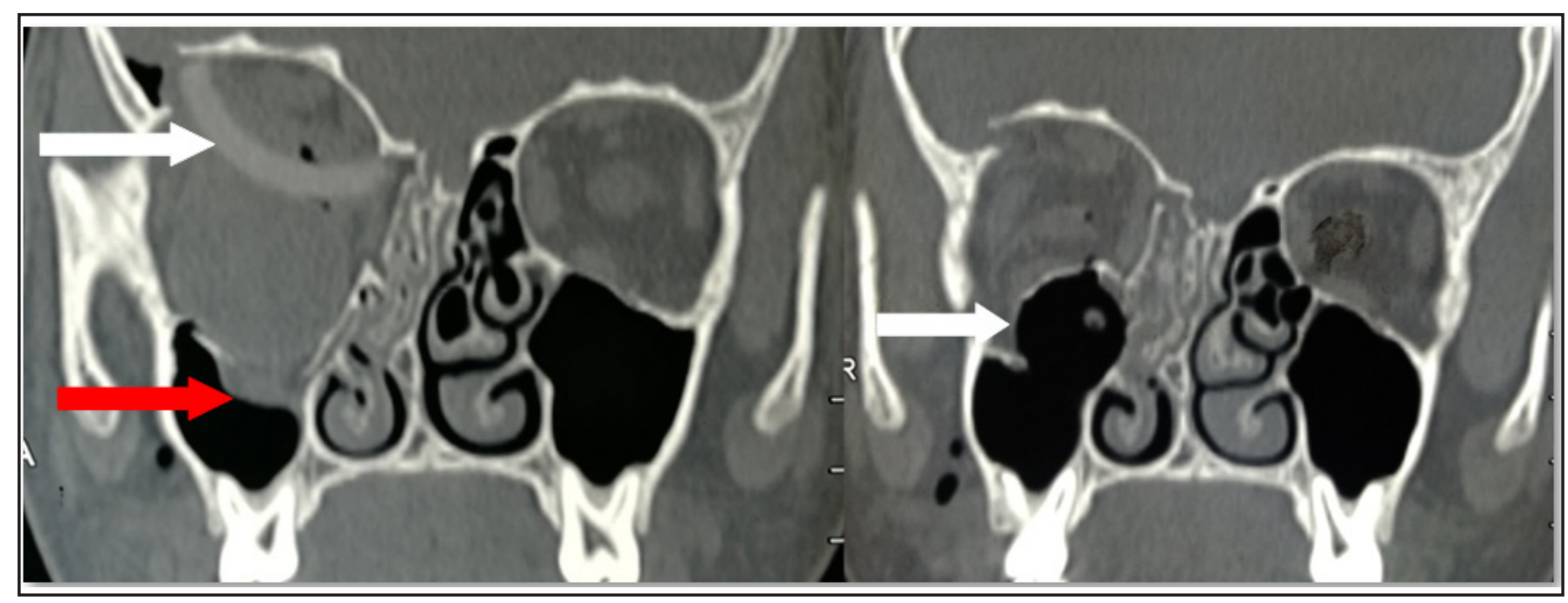

Fig 2: On left, preoperative CT scan of patient showing foreign body in right orbit (white arrow), fracture of right orbital floor, pushing the eyeball downwards (red arrow). On right, immediate postoperative CT scan of patient showing inflated Foley's balloon catheter in right maxillary sinus providing support to right orbital floor (white arrow). 
was done, showing elevation of floor of orbit to near normal level as compared to left side. (Fig. 2)

Postoperative visual assessment of patient was showing presence of pupillary reflex with perception of light and projection of rays present. There was partial movement of eyeball towards medial and inferior aspect during extraocular muscle testing. Movements of lateral and superior rectus muscles were not present. Foleys catheter was removed on postoperative day 7 , eyelid sutures were removed on postoperative day 10 and patient was discharged. The visual acuity was improved to finger counting from 6 feet distance at the time of discharge. (Fig. 1) Lateral and superior movements of eyeball were present 3 weeks after discharge. Currently the patient is under follow up since last 15 months with steady improvement in vision and eyeball movements present in all directions.

\section{Discussion}

Ocular injury is a major cause of treatable visual impairment and blindness. Incidentally, the monthly distribution of ocular trauma often corresponds to the festivals of Holi and Diwali in India. Holi is usually celebrated in March and Diwali in October/ November.1 $5 \%$ of all blindness occurs due to direct result of trauma. According to an estimate under the WHO program for the prevention of blindness, the incidence of open globe injuries in the world is about 2 lakh cases per year which were largely preventable. ${ }^{2}$ Males are more frequently injured than females. ${ }^{3,4}$ Children are more commonly injured than adults due to their curiosity and under developed motor skills. Nearly $90 \%$ eye injuries can be prevented by relatively simple measures. ${ }^{5}$ Though extensive literature is available on ocular trauma and its management, multispecialty management has not been recorded frequently because of rare occurrence of such an extensive trauma and lack of public access to tertiary referral centers. Conservative management of isolated orbital floor fracture is a recognized entity. ${ }^{6}$

Use of multiple specialties of expertise in tertiary referral center is required to deal with such rare case along with a customized approach, as each case is different in presentation. Prompt and effective management with removal of foreign body at the earliest is required to

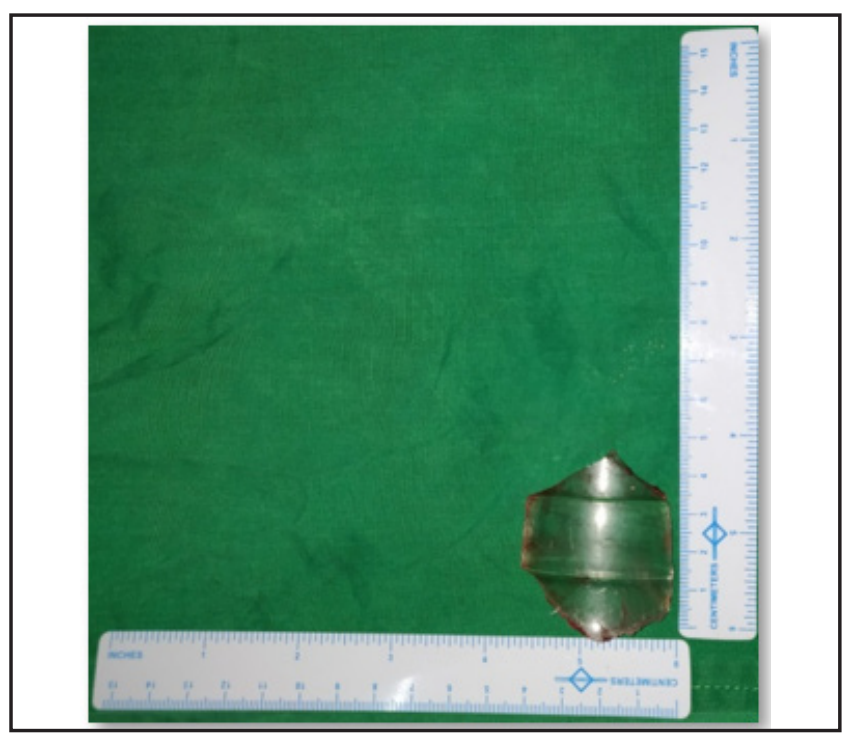

Fig 3: Foreign body specimen

prevent development of complications such as blindness, intraorbital and intracranial infections. Public education about health safety during festival times is a must.

To conclude, the above case has been presented to highlight the effectiveness of a multidisciplinary approach in a tertiary referral center in management of such a rare and complicated case.

\section{References}

1. Ranjan A, Islam MN, Saha M (Dutta Chowdhury). Causes and Clinical Profile of Cases of Ocular Trauma Attending a Tertiary Medical Center. Int J Sci Stud. 2017; 5(3):134-8

2. Sorsby A. Injury. In: Modern Ophthalmology. 22nd ed. London: Butterworth; 1972; 429

3. Khan MD, Mohammed S, Islam ZU, Khattyak MN. An 11 year review of ocular trauma in North West frontier Province of Pakistan. Pakistan Journal of Ophthalmology 1991;7:15-8

4. Kuhn F. Epidemiology of ocular trauma. In: Kuhn F, Morris R, Mester V, Witherspoon D, editors. Ocular Traumatology. Berlin, Heidelberg: Springer-Verlag; 2005; 47-77

5. Jethani J, Vijayalaxmi P. Child: Eye Injuries or causes or prevention and control, public health, health education. India Community Eye Health J Indian Suppl. 2005; 18:58-60

6. Hung J, Poon KJ, Teo NMH, Hing ECH, et al. Conservative management of isolated orbital floor fractures: a retrospective review in a single institution. Int J Oral Maxillofac Surg. 2017; 46 Suppl. DOI. http://dx.doi.org/10.1016/j.ijom.2017.02.881. 